\title{
TRANSFERENCIA DE INMUNIDAD PASIVA EN BUCERRAS Y BUCERROS Y SU INFLUENCIA EN LA ETAPA DE PRE-DESTETE ${ }^{1}$
}

\author{
Beatriz Cáseres-Alvarez², Jorge Alberto Elizondo-Salazar ${ }^{2}$
}

\section{RESUMEN}

Transferencia de inmunidad pasiva en bucerras $y$ bucerros y su influencia en la etapa de pre-destete. El objetivo del presente estudio fue determinar el estado inmunológico de bucerras y bucerros, en una finca dedicada a la explotación bufalina en la región Huetar Norte de Costa Rica. Los datos presentados corresponden a medidas de proteína sérica total obtenidas durante el periodo comprendido entre agosto del 2010 y febrero del 2011, en la provincia de Alajuela, Costa Rica. En dicho lapso de tiempo, la finca fue visitada semanalmente y se tomaron muestras de sangre por venopunción yugular en 116 bucerros (60 hembras y 56 machos) con edades entre 1 y 7 días. Para fines del presente estudio, se consideró una falla en la adquisición de inmunidad pasiva cuando la concentración de proteína sérica total (PST) fue menor a 5,5 g/dl. La concentración de PST varió entre 4,2 y $9,8 \mathrm{~g} / \mathrm{dl}$, con un contenido promedio general de $6,9 \mathrm{~g} / \mathrm{dl}$. De todos los animales evaluados un $12,9 \%$ presentaron niveles inadecuados de inmunidad. Cuando se consideró el sexo de la cría, el 11,7\% de las hembras y el 14,2\% de los machos obtuvieron niveles inadecuados de inmunidad y la concentración de PST no presentó diferencias significativas para hembras y machos (6,9 y 6,8 g/dl, respectivamente). Los bucerros que presentaron una transferencia de inmunidad pasiva adecuada lograron mayor peso vivo durante el segundo y tercer mes de edad con respecto a aquellos machos que tuvieron una falla en la transferencia de inmunidad pasiva.

Palabras clave: búfalo de agua, salud animal, calostro en bucerras, perímetro torácico en bucerras.

\begin{abstract}
Passive transfer of immunity in water buffalo calves and its effect before weaning. The objective of this study was to characterize the immune status of water buffalo calves in a commercial buffalo farm in the Northern Huetar region of Costa Rica. The data presented is for total serum protein measurements obtained between August 2010 and February 2011 in the province of Alajuela, Costa Rica. In that period of time, the farm was visited weekly and blood samples were taken by jugular venipuncture from 116 buffalo calves (60 females and 56 males) between 1 and 7 days of age. For the purpose of this study, failure of passive immunity was considered when TSP was less than $5.5 \mathrm{~g} /$ dl. TSP concentration ranged from 4.2 and $9.8 \mathrm{~g} / \mathrm{dl}$, with an overall mean of $6.9 \mathrm{~g} / \mathrm{dl}$. Of all the animals evaluated, $12.9 \%$ had failure of passive transfer of immunity. When considering sex of the calves, $11.7 \%$ of females and $14.2 \%$ of males failed to obtain adequate levels of immunity, but TSP concentration showed no significant differences between female and male calves (6.9 vs. 6,8 g/dl, respectively). Buffalo male calves that had an adequate passive transfer achieved greater live weight during the second and third months of age with respect to those males which showed a failure of passive transfer of immunity.
\end{abstract}

Keywords: water buffalo, animal health, colostrum in buffalo calves, heart girth in buffalo calves.

\footnotetext{
1 Recibido: 14 de febrero, 2013. Aceptado: 28 de octubre, 2013. Inscrito en la Vicerrectoría de Investigación. Proyecto 737-A9-184. Universidad de Costa Rica.

2 Estación Experimental Alfredo Volio Mata. Facultad de Ciencias Agroalimentarias. Universidad de Costa Rica. Costa Rica. beacaseres@ gmail.com, jorge.elizondosalazar@ucr.ac.cr
} 


\section{INTRODUCCIÓN}

La placenta de los rumiantes previene la transferencia de inmunoglobulinas (Igs) séricas de la madre al feto (Nocek et al. 1984, Argüello et al. 2005). Adicionado a ello, el sistema inmune del neonato es inmaduro e incapaz de producir suficientes Igs para combatir infecciones (Sasaki et al. 1983, Da Silva et al. 1993). Consecuentemente, la ingestión de calostro para los rumiantes recién nacidos es de vital importancia, ya que es la vía por la que obtienen los anticuerpos maternales hasta que su propio sistema inmune llegue a ser completamente funcional (Robinson et al. 1988).

La falla en la transferencia de inmunidad pasiva (FTIP) es el término utilizado para referirse a una deficiencia en el paso de las Igs del calostro a la bucerra o al bucerro. Existe una diversidad de métodos para determinar con precisión el estado de la FTIP. Estos métodos incluyen la medición directa de Igs séricas por medio de la inmunodifusión radial u otras pruebas de ELISA (Filteau et al. 2003). También existen otros métodos indirectos para estimar la concentración de Igs como lo es la medición de las proteínas séricas totales (PST) por refractometría, ya que durante la primera semana de vida, los mayores constituyentes de las proteínas en el suero son las inmunoglobulinas provenientes del calostro (Wallace et al. 2006, TrotzWilliams et al. 2008).

En bovinos, se presenta una FTIP cuando la concentración de PST es menor a 5,2 g/dl (Donovan et al. 1998); sin embargo, otros autores consideran que los animales deben presentar concentraciones mayores a 6,0 g/dl (Davis y Drackley 1998).

Una adecuada absorción de Igs depende del periodo de tiempo que transcurre entre el nacimiento y el suministro de calostro; además, de que los bucerros consuman una cantidad suficiente de Igs, lo cual está determinado por la concentración de estas en el calostro y la cantidad consumida de este (Stott et al. 1979a, b y c), y la eficiencia de absorción de Igs en el intestino (Stott y Fellah 1983, Morin et al. 1997).

Si se presenta algún problema en la absorción de Igs, se observará como resultado un aumento en la incidencia de enfermedades y muerte (Nocek et al. 1984, Hancock 1985, Robinson et al. 1988). Diversas investigaciones, también han demostrado que las inmunoglobulinas del calostro, adquiridas por los animales después del nacimiento, pueden tener un efecto sobre el crecimiento y la producción subsecuente (Denise et al. 1989, Faber et al. 2005, Berge et al. 2009). Así por ejemplo, en terneras de lechería, Robinson et al. (1988) demostraron que una adecuada adquisición de inmunidad pasiva afectó significativamente la variación en las ganancias de peso diarias hasta los 180 días de vida.

En Costa Rica no existe ningún tipo de información científica respecto a la transferencia de inmunidad pasiva en búfalos de agua que permita establecer prácticas de manejo correctivas para mejorar la crianza y desarrollo de los mismos en caso de existir algún problema, ni que relacione la adquisición de inmunidad pasiva con el crecimiento de los animales. El objetivo de la presente investigación fue determinar el estado inmunológico de bucerras y bucerros, y su efecto sobre el crecimiento en la etapa de pre-destete en una finca dedicada a la explotación bufalina en la región Huetar Norte de Costa Rica.

\section{MATERIALES Y MÉTODOS}

\section{Toma de muestras y evaluación de la transferencia de inmunidad pasiva}

Los datos presentados en este estudio corresponden a medidas de proteína sérica total obtenidas durante el periodo comprendido entre agosto del $2010 \mathrm{y}$ febrero del 2011 en una finca comercial en el poblado de Mónico, perteneciente al distrito de Buenavista, cantón de Guatuso de la provincia de Alajuela. En dicho lapso de tiempo, la finca fue visitada semanalmente y se tomaron muestras de sangre por venopunción yugular con el sistema de tubos al vacío sin anticoagulante (tapa roja) en 116 bucerros (60 hembras y 56 machos) con edades entre uno y siete días (Trotz-Williams et al. 2008). La lechería es especializada, es decir el ordeño se hace de forma mecánica en sala de ordeño. Al nacimiento, a las bucerras y bucerros se les permite permanecer todo el primer día con sus respectivas madres para que puedan consumir el calostro. Posteriormente, y hasta los tres meses de edad, los animales permanecen en potreros y todos los días se alimentan de búfalas nodrizas y se suplementan con $300 \mathrm{~g}$ de alimento balanceado. Las razas de las búfalas son Mediterránea, Murrah, Jafarabadi y sus respectivos cruces. Todas las búfalas en el periodo pre y post-parto permanecieron bajo un sistema de pastoreo continuo. 
Las muestras de sangre se manejaron de acuerdo al procedimiento descrito por Johnson et al. (2007), donde estas fueron refrigeradas durante la noche a $4^{\circ} \mathrm{C}$, posteriormente se centrifugaron a $3000 \mathrm{rpm}$ durante $15 \mathrm{minu}-$ tos para separar la fracción sérica. En seguida, la concentración de PST se determinó utilizando un refractómetro de mano (Atago Master-Sur/N $\alpha$, Bellevue, WA).

En ganado de leche, Donovan et al. (1998) han establecido que las terneras presentan una falla en la adquisición de inmunidad pasiva cuando la concentración de PST es menor a 5,2 g/dl; sin embargo, otros autores consideran que las terneras deben presentar concentraciones mayores a 6,0 g/dl (Davis y Drackley 1998). Por lo tanto, para fines del presente estudio, debido a la falta de información disponible, se consideró una falla en la adquisición de inmunidad pasiva cuando la concentración de PST fue menor a 5,5 g/dl.

\section{Pesaje de los animales}

Con la finalidad de relacionar la transferencia de inmunidad pasiva con las ganancias de peso y aumentos de tamaño, las bucerras y bucerros se pesaron al nacimiento y se tomaron las medidas de su circunferencia torácica, además se midió la altura a la cruz y a la cadera. Estas medidas se volvieron a tomar una vez al mes hasta que los animales cumplieron tres meses de edad, momento en que fueron destetados. Las medidas las realizó siempre la misma persona y a la misma hora del día para disminuir el error experimental.

\section{Análisis estadístico}

Para analizar los datos estadísticamente, los recién nacidos se agruparon por sexo, número de parto de la madre y concentración de PST.

Los datos se analizaron por medio del procedimiento MIXED de SAS (SAS Institute 2004). Para determinar la significancia de los efectos del sexo, el número de lactancia de la madre, y la concentración de PST, se consideró al animal como la variable aleatoria y las medidas de peso, circunferencia torácica, altura a la cruz y a la cadera al nacimiento, se utilizaron como covariables. La comparación entre medias se realizó mediante la prueba de Waller-Duncan $(\mathrm{P}<0,05)$. También se generó estadística descriptiva, para determinar la proporción de animales con una FTIP, según el número de lactancia de la madre y el sexo de la cría.
Los datos de peso y perímetro torácico se utilizaron en un modelo cuadrático y lineal para realizar un análisis de correlación simple de Pearson (r), para determinar el grado de asociación entre la variable independiente (perímetro torácico) con la variable dependiente (peso) y poder generar así una ecuación de regresión para la finca, para estimar el peso de los animales a partir del perímetro torácico.

\section{RESULTADOS Y DISCUSIÓN}

\section{Estado inmunológico}

La concentración de PST en bucerras y bucerros con edades entre uno y siete días de nacidos varió entre 4,2 y $9,8 \mathrm{~g} / \mathrm{dl}$, con un promedio general de $6,9 \mathrm{~g} / \mathrm{dl}$. De todos los animales evaluados un 12,9\% (15/116) presentaron falla en la transferencia de inmunidad pasiva (FTIP).

Estos valores son muy semejantes a los reportados por Da Silva et al. (1993) quienes obtuvieron concentraciones de PST entre 6,47 y 8,36 g/dl en bucerras calostradas. Los autores encontraron en búfalos, que al igual que en bovinos, la absorción de proteínas calostrales disminuye considerablemente después de las 24 horas de edad, por lo que concluyeron que el asegurar un consumo temprano de calostro es de gran importancia para que los bucerros y bucerras puedan obtener una adecuada inmunidad pasiva. En otro estudio, Singh y Ahuja (1993) determinaron concentraciones de PST de 4,62 g/dl entre las 31 y 36 horas de vida en bucerras calostradas.

Los resultados obtenidos en el presente trabajo son muy alentadores, pues indican que 9 de cada 10 bucerras y bucerros adquieren una adecuada inmunidad pasiva. Esto es de suma importancia, ya que una baja concentración de Igs en el suero sanguíneo está directamente relacionada con un aumento en la incidencia de enfermedades y muerte (Nocek et al. 1984, Hancock 1985, Robinson et al. 1988).

Estos valores contrastan drásticamente con aquellos reportados para terneras y terneros de lecherías en Canadá y Costa Rica, donde 37,1 y $31,8 \%$, respectivamente, de animales evaluados presentaron una FTIP (Trotz-Williams et al. 2008, Sánchez et al. 2012).

La literatura provee abundante información respecto a los factores que afectan la concentración sérica 
de Igs en animales jóvenes, siendo los más importantes la edad a la que se le ofrece la primera toma de calostro y la masa de Igs ingerida, que a su vez está determinada por el volumen de calostro ofrecido y la concentración de Igs en el mismo (Elizondo 2007).

\section{Sexo de la cría}

La concentración de PST determinada en los animales, sugiere que el 11,7\% (7/60) de las hembras y el $14,2 \%(8 / 56)$ de los machos obtuvieron niveles inadecuados de inmunidad, pero no hubo una diferencia estadísticamente significativa $(\mathrm{P}>0,05)$ para hembras y machos (6,9 y 6,8 g/dl, respectivamente).

En este aspecto, un estudio realizado por Ibrahim y Lemma (2009) determinó que el contenido de PST difirió significativamente entre hembras $(6,8 \mathrm{~g} / \mathrm{dl})$ y machos $(6,4 \mathrm{~g} / \mathrm{dl})$.

Es común encontrar diferencias de este tipo pues un número de investigaciones indican que el sexo de la cría tiene una influencia en la transferencia de inmunidad pasiva, donde por lo general los machos presentan menores concentraciones de PST con respecto a las hembras, debido a que por su mayor tamaño al nacimiento presentan un mayor volumen de plasma sanguíneo que afecta la concentración de proteínas (Quigley et al. 1998, Quigley y Drewry 1998).

\section{Número de parto de la madre}

En el presente estudio se encontraron diferencias significativas $(\mathrm{P}<0,05)$, especialmente en aquellos animales nacidos de madres de segundo y quinto parto (Cuadro 1). Los animales nacidos de madres de segundo parto, presentaron mayores concentraciones de PST cuando se comparan con aquellos nacidos de madres de cinco y más partos. Con base en las variables estudiadas en el presente estudio, es difícil ofrecer una explicación a este hecho, pero estos resultados pudieron responder a un mayor desarrollo de la glándula mamaria o a una ubre colgante en las madres de cinco o más partos que limita la accesibilidad de las crías, por lo que se les dificulta la ingesta de calostro, cuando se comparan con las madres de segundo parto.

\section{Peso y crecimiento de los animales}

Con el fin de determinar si existe alguna asociación entre la adquisición de inmunidad pasiva y el peso vivo de los animales, las crías se agruparon por sexo y edad. En este sentido, no se encontraron diferencias significativas $(\mathrm{P}>0,05)$ para las bucerras; sin embargo, para los bucerros se pudo determinar una diferencia en el segundo y tercer mes de edad, pudiéndose demostrar que los animales con una transferencia de inmunidad pasiva adecuada lograron ganar mayor peso que aquellos machos que tuvieron una FTIP (Cuadro 2).

En un estudio similar, Mastelone et al. (2009) encontraron una asociación lineal significativa $(\mathrm{P}<0,05)$ entre la adquisición de inmunidad pasiva y el peso vivo de los bucerros a los 30 días de edad, igualmente entre la ganancia diaria de peso hasta los 30 días de edad.

Con respecto a las medidas estructurales de crecimiento, no se encontraron diferencias significativas

Cuadro 1. Concentración de proteínas séricas totales (PST) (g/dl) en bucerras y bucerros, de acuerdo al número de parto de la madre, en Guatuso de Alajuela, Costa Rica. 2011.

\begin{tabular}{|c|c|c|c|c|c|c|c|c|c|}
\hline \multirow[b]{2}{*}{ Parto } & \multicolumn{3}{|c|}{ Bucerras } & \multicolumn{3}{|c|}{ Bucerros } & \multicolumn{3}{|c|}{ Total } \\
\hline & n* & PST & EEM** & $\mathbf{n}$ & PST & EEM & $\mathbf{n}$ & PST & EEM \\
\hline 1 & 16 & $6,5 b$ & 0,3 & 14 & $7,1 \mathrm{ab}$ & 0,3 & 30 & $6,8 \mathrm{ab}$ & 0,2 \\
\hline 2 & 10 & $7,6 \mathrm{a}$ & 0,4 & 8 & $7,4 \mathrm{a}$ & 0,4 & 18 & $7,5 \mathrm{a}$ & 0,3 \\
\hline 3 & 15 & $7,1 \mathrm{ab}$ & 0,3 & 15 & $6,8 \mathrm{ab}$ & 0,3 & 30 & $6,9 \mathrm{ab}$ & 0,2 \\
\hline 4 & 9 & $6,9 \mathrm{ab}$ & 0,4 & 8 & $6,7 \mathrm{ab}$ & 0,4 & 17 & $6,8 \mathrm{ab}$ & 0,3 \\
\hline$\geq 5$ & 10 & $6,3 b$ & 0,4 & 11 & $6,3 b$ & 0,3 & 21 & $6,3 b$ & 0,2 \\
\hline
\end{tabular}

* $\mathrm{n}=$ número de animales.

** EEM = Error estándar de la media.

Letras diferentes en una misma columna difieren estadísticamente $(\mathrm{P}<0,05)$. 
Cuadro 2. Peso $(\mathrm{kg})$ de las crías de búfalas, categorizados por sexo y edad de acuerdo a la adquisición de inmunidad pasiva en Guatuso de Alajuela, Costa Rica. 2011.

\begin{tabular}{lcccccc}
\hline & \multicolumn{2}{c}{ Bucerras } & & \multicolumn{2}{c}{ Bucerros } \\
\cline { 2 - 3 } \cline { 5 - 6 } Edad & No adecuada & Adecuada & & No adecuada & Adecuada \\
\cline { 1 - 2 } \cline { 5 - 6 } Nacimiento & 38,2 & 38,2 & & 37,1 & 38,1 \\
1er mes & 50,6 & 53,2 & & 50,9 & 52,8 \\
2do mes & 69,2 & 68,6 & & $63,8 \mathrm{~b}$ & $71,7 \mathrm{a}$ \\
3er mes & 91,3 & 88,1 & & $83,2 \mathrm{~b}$ & $93,7 \mathrm{a}$ \\
EEM* & 3,8 & 1,4 & & 3,5 & 1,5 \\
\hline
\end{tabular}

*EEM = Error estándar de la media.

Letras diferentes en una misma fila difieren estadísticamente $(\mathrm{P}<0,05)$.

$(\mathrm{P}>0,05)$ cuando se quiso relacionar el perímetro torácico, la altura a la cruz y de cadera con la transferencia de inmunidad pasiva (Cuadro 3); esto pudo deberse a la poca cantidad de animales que presentaron una FTIP (solamente el 12,9\% de todos los animales evaluados). Sin embargo, se considera importante presentar los datos para que puedan servir como parámetro de referencia para futuros trabajos con búfalos.

Cuadro 3. Perímetro torácico $(\mathrm{cm})$, altura a la cruz $(\mathrm{cm})$ y altura a la cadera $(\mathrm{cm})$ de las crías de búfalas, de acuerdo a la adquisición de inmunidad pasiva, categorizados por sexo y edad, en Guatuso de Alajuela, Costa Rica. 2011.

\begin{tabular}{|c|c|c|c|c|}
\hline & \multicolumn{2}{|c|}{ Bucerras } & \multicolumn{2}{|c|}{ Bucerros } \\
\hline & No adecuada & Adecuada & No adecuada & Adecuada \\
\hline \multicolumn{5}{|c|}{ Perímetro torácico } \\
\hline Nacimiento & 80,3 & 80,4 & 80,6 & 80,5 \\
\hline 1er mes & 88,5 & 89,2 & 90,2 & 89,5 \\
\hline 2do mes & 99,8 & 97,1 & 96,6 & 96,6 \\
\hline 3er mes & 111,9 & 106,3 & 106,1 & 106,9 \\
\hline EEM & 2,0 & 0,7 & 1,9 & 0,6 \\
\hline \multicolumn{5}{|c|}{ Altura a la cruz } \\
\hline Nacimiento & 71,7 & 72,1 & 71,4 & 72,2 \\
\hline 1er mes & 78,0 & 77,4 & 77,7 & 77,2 \\
\hline 2do mes & 83,2 & 82,2 & 83,0 & 82,2 \\
\hline 3er mes & 89,1 & 88,6 & 86,4 & 87,4 \\
\hline EEM & 1,0 & 0,4 & 0,9 & 0,4 \\
\hline \multicolumn{5}{|c|}{ Altura a la cadera } \\
\hline Nacimiento & 75,6 & 75,7 & 75,1 & 75,6 \\
\hline 1er mes & 80,6 & 81,6 & 82,4 & 82,4 \\
\hline 2do mes & 86,8 & 86,5 & 87,8 & 88,0 \\
\hline 3er mes & 93,1 & 92,8 & 93,8 & 93,5 \\
\hline EEM* & 1,0 & 0,4 & 1,0 & 0,4 \\
\hline
\end{tabular}

*EEM = Error estándar de la media. 


\section{Ecuaciones de regresión para estimar el peso a par- tir del perímetro torácico}

El monitoreo del crecimiento de los animales en cualquier explotación pecuaria, permite determinar si las metas de un buen programa de desarrollo animal se están alcanzado. El peso vivo de los animales es la expresión más confiable para medir dicho crecimiento y refleja la disponibilidad de alimentos para expresar su potencial genético (Álvarez et al. 1999). El método más preciso para determinar el peso corporal es utilizar una romana calibrada. Sin embargo, muchas fincas carecen de ella, por lo que la determinación de una ecuación de predicción que permita relacionar la medida del perímetro torácico con el peso de los animales, podría brindar una herramienta de gran utilidad, para que así se pueda evaluar el crecimiento de los animales con el fin de tomar decisiones tanto en los programas de alimentación como en el manejo sanitario y reproductivo de los animales.

Se observó un valor de $\mathrm{r}=0,90$ altamente significativo $(\mathrm{P}<0,01)$ en la relación entre el peso vivo y el perímetro torácico de los animales, lo que indica que estas variables están altamente asociadas y confirma que un cambio en el peso vivo del animal puede detectarse a una variación en el perímetro torácico (Figura 1). Debido a que los coeficientes de determinación fueron muy similares para el modelo cuadrático como para el lineal, se consideró trabajar con el modelo más simple, de manera que pueda ser utilizado más fácilmente por los productores. Las ecuaciones de regresión obtenidas fueron:

Considerando todos los animales:

$\mathrm{Y}=0,151 \mathrm{X}+27,461 \quad \mathrm{r}^{2}=0,82$

Considerando solamente las bucerras:

$\mathrm{Y}=0,1428 \mathrm{X}+28,83 \quad \mathrm{r}^{2}=0,81$

Considerando solamente los bucerros:

$\mathrm{Y}=0,1603 \mathrm{X}+25,797 \quad \mathrm{r}^{2}=0,82$

Donde $\mathrm{Y}=$ Peso del animal $(\mathrm{kg})$ y $\mathrm{X}=$ Perímetro torácico $(\mathrm{cm})$.

En un estudio llevado a cabo en Costa Rica en ganado Zebú, para evaluar la relación existente entre el perímetro torácico y el peso corporal de animales en crecimiento, en una muestra de 2105 animales, Garro y Rosales (1996) encontraron diferentes ecuaciones cuyo coeficiente de determinación $\left(\mathrm{R}^{2}\right)$ varió entre 0,83 y 0,98 . Mahecha et al. (2002a, 2002b) obtuvieron resultados similares en el ganado bovino de la raza Lucerna en Colombia, con un coeficiente de determinación $\left(\mathrm{R}^{2}\right)$ de 94,28 $(\mathrm{P}<0,05)$.

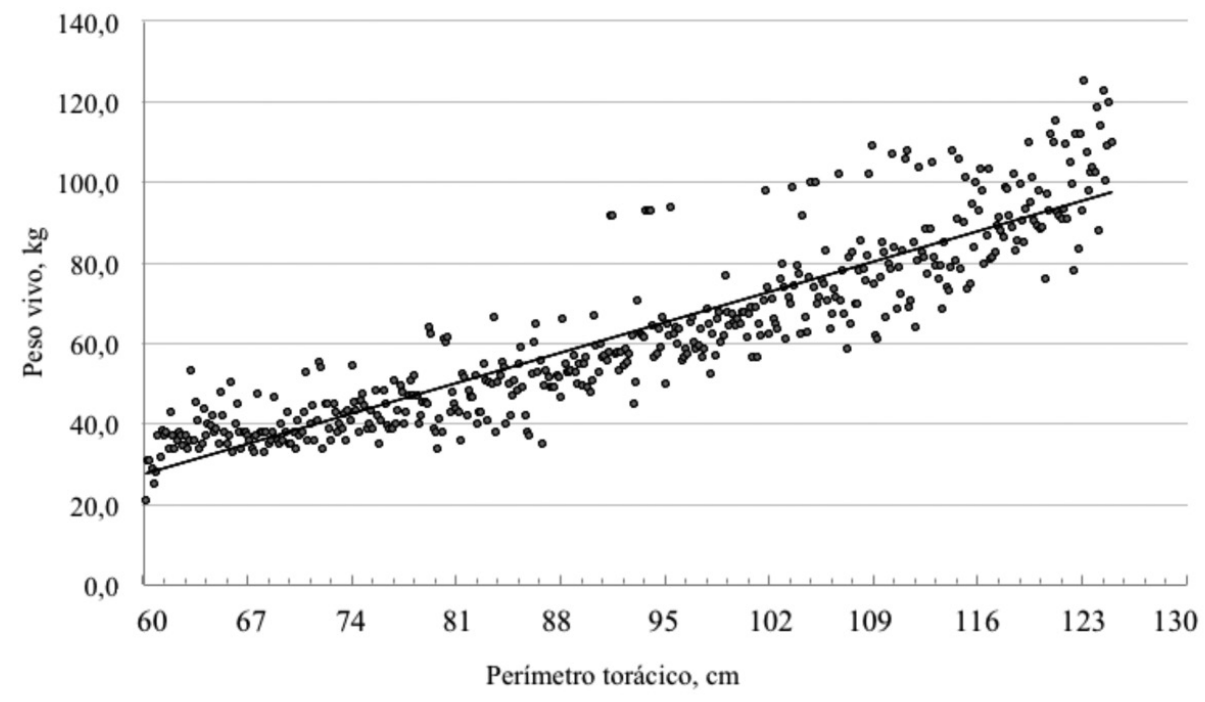

Figura 1. Relación entre el perímetro torácico $(\mathrm{cm})$ y el peso vivo $(\mathrm{kg})$ en bucerras y bucerros de 0 a 3 meses de edad, en Guatuso de Alajuela, Costa Rica. 2011. 
Nuestros resultados indican que bucerros que lograron una adecuada inmunidad pasiva pueden mejorar los índices de crecimiento durante los primeros tres meses de edad, sin embargo, más estudios son necesarios para corroborar los resultados obtenidos.

Pese a que los valores obtenidos en la FTIP son inferiores a los reportados en terneras de lechería, es necesario establecer prácticas de manejo del calostro que permitan minimizar el riesgo de bucerras y bucerros con una transferencia de inmunidad pasiva inadecuada en esta y otras explotaciones bufalinas de nuestro país.

\section{AGRADECIMIENTOS}

Los autores desean expresar su más sincero agradecimiento al dueño de la finca El Porvenir y a sus gentiles colaboradores por habernos permitido llevar a cabo el presente estudio.

\section{LITERATURA CITADA}

Álvarez, RZ; Vaccaro, L; Vaccaro, R; Verde, O; Ríos, L; Mejías, H. 1999. Estimación de pesos de becerros de doble propósito a partir de mediciones corporales. Rev. Cien. FCV-LUZ 9:502-507.

Argüello,A; Castro, N; Capote, J. 2005. Short communication: evaluation of a color method for testing immunoglobulin $\mathrm{G}$ concentration in goat colostrum. J. Dairy Sci. 88:1752-1754.

Berge, AC; Besser, TE; Moore, DA; Sischo, WM. 2009. Evaluation of the effects of oral colostrums supplementation during the first fourteen days on the health and performance of preweaned calves. J. Dairy Sci. 92:286-295.

Da Silva, MC; Queiroz, W; Didonet, LH; Gomez, VW. 1993. Colostrum and serum protein levels in water buffaloes. Pesq. Agropec. Bras. 28:751-757.

Davis, CL; Drackley, JK. 1998. The development, nutrition, and management of the young calf. Ames, Iowa, USA, Iowa State University Press. 339 p.

Denise, SK; Robison, JD; Stott, GH; Armstrong, DV. 1989. Effects of passive immunity on subsequent production in dairy heifers. J. Dairy Sci. 72:552-554.

Donovan, GA; Dahoo, IR; Montgomery, DM; Bennett, FL. 1998. Associations between passive transfer immunity and morbidity and mortality in dairy heifers in Florida, USA. Prevent. Vet. Med. 34:31-46.

Elizondo, JA. 2007. Alimentación y manejo del calostro en el ganado de leche. Agronomía Mesoamericana 18(2):271-281.

Faber, SN; Faber, NE; McCauley, TC; Ax, RL. 2005. Effects of colostrum ingestion on lactational performance. The Professional Animal Scientist 21(5):420-425.

Filteau, V; Bouchard, E; Fecteau, G; Dutil, L; Dutremblay, D. 2003. Health status and risk factors associated with failure of passive transfer of immunity in newborn beef calves in Quebec. Can. Vet. J. 44:907-913.

Garro, JM; Rosales, LR. 1996. Relación entre el peso corporal y el perímetro torácico en ganado cebuino en crecimiento en Costa Rica. Agronomía Costarricense 20(2):113-123.

Hancock, DD. 1985. Assessing efficiency of passive immune transfer in dairy herds. J. Dairy Sci. 68:163-183.

Ibrahim, A; Lemma, A. 2009. Relationships between serum protein concentration and passive transfer of immunity, morbidity and mortality of dairy calves in market oriented urban dairy farms. Revue Méd. Vét. 160(8-9):394-399.

Johnson, JL; Godden, SM; Molitor, T; Ames, T; Hagman, D. 2007. Effects of feeding heat-treated colostrum on passive transfer of immune and nutritional parameters in neonatal dairy calves. J. Dairy Sci. 90: 5189-5198.

Mahecha, L; Angulo, J; Manrique, LP. 2002a. Estudio bovinométrico y relaciones entre medidas corporales y el peso vivo en la raza Lucerna. Rev. Col. Cienc. Pec.15:80-87.

Mahecha, L; Angulo, J; Manrique, LP. 2002b. Predicción del peso vivo a través del perímetro torácico en la raza bovina Lucerna. Rev. Col. Cienc. Pec. 15:88-91.

Mastelone, V; Massimini, G; Pero, M; Zicarelli, G; Sattar, A; Cestaro, A; Lombardi, P; Britti, D; Zicarelli, F; Vecchio, D. 2009. Passive transfer status as a significant source of variationon growth performance in buffalo calves. Pakistan J. Zool. Suppl. Ser. 9:147149.

Morin, DE; McCoy, GC; Hurley, WL. 1997. Effects of quality, quantity, and timing of colostrum feeding and addition of dried colostrum supplement on immunoglobulin $\mathrm{G}_{1}$ absorption in Holstein bull calves. J. Dairy Sci. 80:747-753.

Nocek, JE; Braund, DG; Warner, RG. 1984. Influence of neonatal colostrums administration, immunoglobulin, 
and continued feeding of colostrums on calf gain health, serum protein. J. Dairy Sci. 67:319-333.

Quigley, JD; Drewry, JJ. 1998. Nutrient and immunity transfer from cow to calf pre- and postcalving. J. Dairy Sci. 81:2779-2790.

Quigley, JD; Drewry, JJ; Martin, KR. 1998. Estimation of plasma volume in Holstein and Jersey calves. J. Dairy Sci. 81:1308-1312.

Robison, JD; Stott, GH; DeNise, SK. 1988. Effects of passive immunity on growth and survival in the dairy heifer. J. Dairy Sci. 71(5):1283-1287.

Sánchez, J; Elizondo, JA; Arroyo, G. 2012. Estado inmunológico de terneras y terneros de lechería en la región Huetar Norte de Costa Rica. Año I. Agronomía Mesoamericana 23(2):321-327.

SAS (SAS Institute Inc, US). 2004. SAS/STAT 9.1 User`s guide. Version 9.1 Cary, N.C. 5121 p.

Sasaki, M; Davis, CL; Larson, BL. 1983. Immunoglobulin $\mathrm{IgG}_{1}$ metabolism in new born calves. J. Dairy Sci. 60: 623-626.

Singh, A; Ahuja, SA. 1993. Individual variation on the composition of colostrum and absorption of colostral antibodies by the precolostral buffalo calf. J. Dairy Sci. 76:1148-1156.

Stott, GH; Marx, DB; Menefee, BE; Nightengale, GT. 1979a. Colostral immunoglobulin transfer in calves. I. Period of absorption. J. Dairy Sci. 62:1632-1638.

Stott, GH; Marx, DB; Menefee, BE; Nightengale, GT. 1979b. Colostral immunoglobulin transfer in calves. II. The rate of absorption. J. Dairy Sci. 62:1766-1773.

Stott, GH; Marx, DB; Menefee, BE; Nightengale, GT. 1979c. Colostral immunoglobulin transfer in calves. III. Amount of absorption. J. Dairy Sci. 62:1902-1907.

Stott, GH; Fellah, A. 1983. Colostral immunoglobulin absorption linearly related to concentration for calves. J. Dairy Sci. 66:1319-1328.

Trotz-Williams, LA; Leslie, KE; Peregrine, AS. 2008. Passive immunity in Ontario dairy calves and investigation of its association with calf management practices. J. Dairy Sci. 91: 3840-3849.

Wallace, MM; Jarvie, BD; Perkins, NR; Leslie, KE. 2006. A comparison of serum harvesting methods and type of refractometer for determining total solids to estimate failure of passive transfer in calves. Can. Vet. J. 47: 573-575. 